\title{
Le jeu du marché et le poids du domaine public en aménagement forestier au Québec
}

\author{
par Luc Bouthillier ${ }^{1}$
}

\section{Résumé}

Ce texte part d'un questionnement sur le fonctionnement des forces du marché dans le secteur forestier québécois. Pour mieux comprendre la situation, on y effectue des comparaisons avec la Suède, le Royaume-Uni et les États-Unis. L'auteur y constate que le nouveau régime forestier du Québec pourrait contenir des éléments de solution aux problèmes d'un domaine public trop vaste et non géré selon un mode entrepreneurial.

Mots clés: Allocation, domaine public, tarification

\begin{abstract}
This article comes from a concern about the impact of the market system on Quebec's forest sector. Comparisons between Sweden, the United Kingdom, the United States and Quebec allow a clear picture of the situation. It is shown that the new Quebec forest system could bring elements of solution. This system has to deal with the problem of an oversized public domain and with the lack of economical foundation for the forest management program it promotes.
\end{abstract}

Key words: Forest allotment, public domain, stumpage

\section{Une entrée en matière}

Laisser libre cours aux forces du marché en foresterie constitue un jeu plein de rebondissements. Ce jeu est vaste, débordant de promesses mais aussi de difficultés. Il ouvre des perspectives pour solutionner plusieurs problèmes. Il permet d'envisager une manière rationnelle d'allouer les bois des forêts québécoises. Il n'en faut donc pas plus pour qu'on $\mathrm{y}$ porte une attention toute spéciale.

À l'occasion de l'implantation du nouveau régime forestier adopté au Québec, l'examen du marché en tant que système automatique d'allocation des ressources est particulièrement pertinent. Mentionnons tout de suite que notre démarche consistera à considérer des expériences que d'autres pays ont faites en la matière. C'est un peu comme si a priori, nous admettions que l'allocation des bois se pratique encore de manière arbitraire au Québec. Conséquemment, il ne nous resterait plus qu'à aller voir ailleurs comment les forces du marché se manifestent pour réaliser qu'elles pourraient jouer de façon analogue au Québec.

Inutile d'insister sur les doutes entretenus quant à la vivacité du marché pour la matière ligneuse au Québec. L'absence du marché constitue même un cliché dans le monde forestier québécois. En cours d'exposé, nous tenterons de montrer qu'il s'agit d'une présomption. Les forces du marché sont à l'oeuvre au Québec. Toutefois, elles ne se conforment pas au modèle idéal. Nous proposons donc un tour d'horizon qui mettra en lumière certaines dimensions de la problématique forestière du Québec influençant l'action des forces du marché de façon bien spécifique en termes d'allocation et de tarification de la matière ligneuse. Nous tenterons aussi de rattacher cette démonstration au thème de la conciliation à effectuer entre l'envirennment et l'économie.

\section{Concurrence et dirigisme}

Fer de lance de l'analyse économique, la loi de l'offre et de la demande que sous-entend le phénomène du marché est d'une logique assez simple. Pour une période donnée, plus les quantités demandées sont élevées, plus les prix montent.

\footnotetext{
${ }^{1}$ Professeur assistant à la Faculté de foresterie et de géomatique de
} l'Université Laval.
À l'opposé, plus les quantités offertes sont grandes, plus les prix baissent. Fait saillant du système, la situation d'équilibre entre l'offre et la demande est synonyme d'efficacité. La validité de cette convention est toutefois sujette à des exigences. Contentons-nous d'observer qu'elle est attachée au monde de la concurrence parfaite. Un monde un peu irréel où une foule de vendeurs et d'acheteurs, qui sont pleinement informés des conditions du marché et incapables de contrôler les prix, transigent un bien homogène.

Dans leur compréhension du secteur primaire en foresterie, d'aucuns retiennent la situation de concurrence parfaite pour fonder leur verdict sur la non-existence du marché. Sous l'angle du nombre d'acteurs, le Québec semble de fait se soustraire au marché puisque l'État occupe une place prépondérante comme propriétaire forestier. Cette position déterminante amènerait l'Etat à exercer un dirigisme allant à l'encontre du marché et de la mise en valeur des forêts. Pour certains désirant donner plus de latitude aux forces du marché, il faudrait donc réduire l'emprise de l'État sur la propriété du territoire. Pour d'autres, il suffirait de réécrire la partition confiée à ses mandataires.

En fait, la solution au problème de gestion forestière posé par le poids du territoire placé sous la juridiction de l'État québécois nous semble tenir dans les deux dernières suggestions. La privatisation des terres publiques est de loin la recommandation la plus spectaculaire. D'ailleurs sur la scène mondiale, le démembrement des domaines publics n'est pas un projet nouveau. Épisodiquement, la redistribution des titres de propriété alimente les discussions au sujet d'une meilleure gestion forestière. Parfois, la parole tente de sc faire acte. Les difficultés rencontrées obligent alors à plutôt considérer une redéfinition de la tâche des intervenants publics en production forestière. Prenons par exemple, les cas de la Suède et du Royaume-Uni.

\section{Le domaine public}

\subsection{Le cas suédois}

Après une trentaine d'années de libéralisme pur et dur pendant lesquelles le secteur privé avait fait l'acquisition d'une grande partie des terres de la couronne, la Diète suédoise institue le Domänverket en 1859. Les autorités 
cherchent alors à contrer le mouvement de privatisation. Elles réagissent à une opinion populaire inquiète de l'état de la forêt suédoise. En fait, l'allure générale des massifs forestiers au milieu du $\mathrm{XIX}^{\mathrm{e}}$ siècle laisse croire qu'en Suède, la civilisation est l'ennemie jurée de la forêt (von Hofsten, 1980).

Au début de son histoire, le Domänverket a la responsabilité, à titre d'Office national des forêts, de superviser la gestion des ressources forestières détenues par l'industrie et l'église. Simultanément, il doit garder, protéger et gérer ce qui reste de forêt publique. À cette époque, la superficie administrée en propre par le Domänverket ne couvre que 70000 hectares principalement localisés dans le nord du pays.

Mais avec les années, le domaine public s'agrandit. Par un programme de rachats, les autorités suédoises accroissent graduellement le territoire placé sous l'administration directe de l'organisme public. On récupère ainsi les droits de propriété de plusieurs compagnies en déclin et aussi ceux de petits exploitants agricoles marginaux. Parallèlement, le Domänverket perd son statut d'Office des forêts. Avec le développement de l'administration publique, entre 1905 et 1924, l’organisme se voit déchargé de toutes les responsabilités concernant des forêts autres que publiques. En 1969, le gouvernement suédois lui conferre l'appellation d'entreprise lucrative à caractère industriel et commercial.

Aujourd'hui le Domänverket contrôle plus de dix millions d'hectares dont 40 p. 100 est composé de forêts productives (von Hofsten, 1980). Il détient donc 20 p. 100 de la forêt commerciale suédoise et fournit chaque année de 8 à 10 millions de $\mathrm{m}^{3}$ de bois à prix compétitif. Rappelons qu'en Suède la récolte globale de matière ligneuse atteindrait annuellement 67 millions de $\mathrm{m}^{3}$ (Hermelin, 1986). Observons également que l'industrie forestière suédoise peut consommer toute la matière première qu'on lui rend disponible (von Sidow, 1985). La dernière remarque est importante pour caractériser le marché des bois bruts en Suède. Elle définit un marché affecté par une rareté relative. Celui-ci peut donc absorber à bon prix l'ensemble des quantités offertes.

\subsection{Le cas britannique}

Le Royaume-Uni avec sa Forestry Commission représente aussi un cas d'espèce quant à la soi-disant mainmise de l'État sur une partie du territoire forestier. À entrée du $\mathrm{XX}^{\mathrm{e}}$ siècle, la fière Albion ne dispose plus que d'un maigre million d'hectares de forêts (Johnston et al. 1967). Le pays s'appuie sur le commerce international pour satisfaire ses besoins en bois. Vu les capacités domestiques de production qui sont insuffisantes et les prix internationaux qui défient les prix locaux, l'industrie préfère compter sur l'étranger afin de s'assurer des approvisionnements en matière ligneuse. Les propriétaires forestiers destinent leurs terres vers d'autres genres d'activités ou encore, les laissent à l'abandon.

La grande guerre de 1914-1918 bouleverse cet ordre des choses. Coupé de ses sources externes d'approvisionnement ne bois, le Royaume-Uni assiste à la dilapidation de ses dernières réserves forestières. Pour remédier à la ruine de son patrimoine forestier, le Parlement britannique met en place la Forestry Commission dès 1919. La tâche principale de cet organisme consiste à relancer la production forestière dans les îles britanniques. On lui confie une large proportion du domaine public afin qu'il puisse lui-même amorcer le mouvement. De plus, la Forestry Commission est dotée de moyens physiques et financiers pour inciter les propriétaires privés à lui emboîter le pas.

Concrètement, l'action de la Forestry Commission se traduit par l'enrésinement de zones situées dans le nord ouest de l'Angleterre, le pays de Galles et l'Écosse. Soixante-dix ans après la création de la Forestry Commission, la forêt britannique occupe une superficie de 2,1 millions d'hectares. L'institution publique gère directement 42 p 100 de cette aire (Campbell, 1988). On lui attribue plus de la moitié des 4,9 millions de $\mathrm{m}^{3}$ récoltés au Royaume-Uni en 1987. Bien plus, cette production de matière ligneuse rencontre des critères de rentabilité économique (Price, 1989).

Encore une fois, notons l'importance des quantités demandées vis-à-vis des quantités offertes de matière ligneuse, Au Royaume-Uni, le taux d'autosuffisance en bois atteint tout juste 16 p. 100 pour l'année 1987 (Campbell, 1988). Il faut aussi prendre en considération que la Forestry Commission est perçue comme un outil de développement régional (Price, 1989). En outre, sa mission est intimement lié à la politique agricole du pays. Le gouvernement britannique entend liquider certains surplus de production agricole en détournant l'usage des terres vers la foresterie. Remarquons finalement que depuis 1988, le Parlement de Londres exige de la Forestry Commission qu'elle s'autofinance. Celle-ci se plierait à cette requête en vendant des morceaux de son domaine (Campbell, 1988).

\section{De l'influence et de la dimension du domaine public}

Que dégager de ces deux exemples? D'abord que la concurrence parfaite compose un idéal aidant à comprendre le fonctionnement du marché qui n'a pourtant pas besoin d'elle pour exister dans la réalité du secteur forestier. En effet, l'absence de compétition parfaite se justifie structurellement.

Les besoins en capitaux nécessaires pour faire fonctionner l'industrie manufacturière de produits forestiers sont relativement élevés. Ils dépassent de loin ceux requis pour entrer dans la production de bois. Il peut donc exister un déséquilibre entre les agents économiques impliqués dans le marché de la matière ligneuse. Le nombre de joueurs du côté de l'offre peut facilement excéder celui du côté de la demande. Un domaine public géré par l'État ou son mandataire tire sa raison d'être dans une fonction de balancier visant à corriger ce déséquilibre.

Comme le montrent les expériences suédoises et britanniques, des institutions publiques de gestion forestière peuvent occuper des positions stratégiques sans fausser le jeu du marché. Tant et aussi longtemps que le prix de la matière ligneuse n'est pas fixé arbitrairement et que les quantités demandées de matière ligneuse demeurent élevées par rapport aux capacités de la forêt, les circonstances semblent favorables à la manifestation d'un esprit d'entreprise en production forestière. Les besoins en matière ligneuse sont tels que la production de bois constitue en centre de profits. Des entreprises parapubliques ont donc la partie belle pour catalyser des efforts d'aménagement forestier sur l'ensemble du territoire national.

En d'autres termes dans un contexte québécois, la question consiste à se demander si le marché pour la matière ligneuse 
présente des conditions similaires à celles de la Suède et du Royaume-Uni. Dans l'affirmative, il faudrait réfléchir à la manière dont l'État peut utiliser le poids du domaine public pour intensifier le travail d'aménagement en forêt et permettre à des entrepreneurs de mieux réaliser des occasions de profits.

Pour plusieurs, l'envergure de son domaine public donne à l'État québécois la faculté de dévier du rôle de balanciercatalyseur que nous en attendons. De fait avec 85 p. 100 du territoire forestier productif et accessible de la province, le Québec, à l'instar du Canada, se distingue des autres contrées forestières de l'hémisphère nord (voir Tableau 1). Encore faut-il réaliser que tant en Suède qu'au Royaume-Uni, ce n'est pas l'importance du domaine mais la séparation entre les fonctions de propriétaire et d'administrateur public qui fait la différence. Là-bas, on différencie clairement l'État propriétaire de l'État administrateur. Le Domänverket comme la Forestry Commission composent des entités publiques vouées à la production et non à la police des agents économiques.

\section{L'allocation de la matière ligneuse sur le domaine public}

Le dernier point fournit un élément de départ pour traiter de la question de l'allocation des bois sur les terres publiques. Le degré de la césure entre l'État-puissance publique et l'Étatpropriétaire semble permettre de mieux définir le jeu des forces du marché dans l'attribution des produits du domaine public. Plus la séparation serait marquée, plus l'influence de forces du marché se ferait sentir dans une direction propice à la production forestière quand l'industrie consommatrice est éprouvée par une situation de rareté. L'histoire scandinave permet de rajouter un raffinement à cette affaire (von Hofsten, 1980 et Appelroth, 1984). À la lumière des faits rapportés, le mode d'allocation en forêt publique semble se conformer à un modèle intégrant l'intensité de sylviculture pratiquée (voir figure un).

Le modèle comporte trois volets. Quand le travail en forêt se limite à l'abattage d'une forêt primitive, on assiste à la situation suivante: le droit de couper des arbres sur le domaine public est accordé à des entrepreneurs privés pour de courtes ou moyennes périodes de temps en échange d'une redevance calculée en fonction des besoins fiscaux de l'État concédant. Notons que règle générale, ce dernier est alors en voie de développement. Ensuite, à mesure que l'État spécifie des règles d'exploitation et les fait respecter, on

Tableau 1. Superficie de la forêt productive et superficie du domaine public dans cinq régions de l'hémisphère nord.

\begin{tabular}{lccc}
\hline & $\begin{array}{c}\text { Aire totale } \\
\text { de la forêt } \\
\text { productive }\end{array}$ & $\begin{array}{c}\text { Aire de la } \\
\text { forêt productive } \\
\text { appartenant au } \\
\text { domaine public }\end{array}$ & $\begin{array}{c}\text { Pourcentage } \\
\text { occupé par le } \\
\text { domaine } \\
\text { public }\end{array}$ \\
\cline { 2 - 4 } & \multicolumn{4}{c}{ (millions d'hectares) } \\
\cline { 2 - 4 } Suède & 23,5 & $4,1^{1}$ & $17 \%$ \\
Finlande & 19,7 & 4,7 & $24 \%$ \\
Royaume-Uni & 2,1 & 0,9 & $43 \%$ \\
États-Unis & 195,5 & 36,0 & $18 \%$ \\
Canada & 220,0 & 200,0 & $91 \%$ \\
Québec & 54,6 & 48,0 & $88 \%$ \\
\hline
\end{tabular}

${ }^{1}$ Deux millions d'hectares appartenant à l'Église d'état sont exclus de ce chiffre. passe à la phase suivante: l'aménagement du base. L'objectif des gouvernements est à ce moment dominé par un désir d'affermir la base industrielle de l'espace gouverné. Cette fois, les droits de couper sont acquis pour une plus longue durée par des entrepreneurs généralement intégrés à l'industrie de transformation.

Vient le moment où la forêt apparait comme un facteur limitant pour l'industrie, on entre alors dans la phase d'aménagement intensif. Habituellement, la rareté relative des billes par rapport aux besoins des fabricants de produits forestiers amène le propriétaire public à saisir une occasion de profits dans une telle situation. Il n'est plus question de droits de couper à long terme. On revient au système initial d'allocation à une différence près. Les droits pour récolter des bois sur pied sont octroyés au plus offrant par voie d'enchères publiques. Les agences responsables du domaine public ont même tendance à prendre en main les opérations de récolte pour mieux les intégrer aux activités sylvicoles. Ainsi, elles préfêrent soumettre aux enchères des volumes de matière ligneuse empilés au bord de la route ou encore livrables sur place à l'usine.

Il faut souligner qu'en Scandinavie, les bois du domaine public sont adjugées à des entrepreneurs privés par le mécanisme des enchères depuis la fin du XIX ${ }^{\mathrm{e}}$ siècle. $\mathrm{La}$ situation de rareté physique perceptible à partir de $1850 \mathrm{a}$ permis de passer très rapidement à une phase sylvicole animée par un projet de rendement accru (von Sydow, 1985). $\mathrm{Au} \mathrm{XX} \mathrm{X}^{\mathrm{e}}$ siècle la prise en charge progressive des opérations de récolte par les agences publiques est remarquable. De nos jours, plus de 95 p. 100 de la production ligneuse du Domänverket est adjugée à port de camion (voir figure deux). La même situation se présenterait en Finlande et au Royaume-Uni.

\section{Tarification, sylviculture et propriété.}

Le grand mérite du système des enchères publiques est la formalisation d'une transaction autour de la matière ligneuse, soit en forêt, soit en bordure de route, qui exclut le dirigisme de l'État sur les prix. Pour le gros producteur de bois que constitue une agence de gestion du domaine public, la connaissance des intrants et de leur coût permet de fixer une mise à prix qui sera modulée au gré d'une transaction effectuée selon les règles du libre marché. Par la même occasion, la recherche d'une tarification adéquate des bois sur pied perd sa raison d'être. Quant à elle, l'intégration des travaux de récolte à la fonction de production de matière ligneuse permet une meilleure distinction entre les producteurs de bois et les industriels de la transformation. $\mathrm{La}$ constitution d'un marché pour la filière forêt-bois est consolidée.

Chez nos voisins américains, la matière ligneuse des National Forests est écoulée par voie d'enchères publiques. Mais contrairement à la plupart de ses homologues européens, le U.S. Forest Service privilégie la vente de bois debout. Les récentes controverses au sujet de ces ventes apparaissent valider le modèle développé plus haut et éclairent la problématique québécoise. On reproche au U.S. Forest Service de laisser aller les bois à des prix inférieurs à leur coût de production (Emerson et al. 1984). Le mécanisme des enchères sousestimerait la valeur des tiges. L'explication du litige semble résider dans la nature des National Forests et du corps public responsable de leur gestion. 
Figure 1. Modèle de l'encadrement du domaine public en regard de la rareté relative de matière

allocation ligneuse.
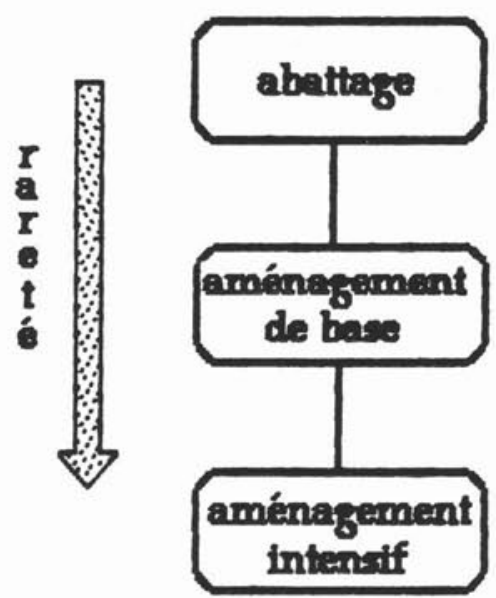

tarification

besoins

fiscaux

du

gouvernement

contrat

à long

développement

de la base

induatrielle

production

profitable

de

matière

ligneuve

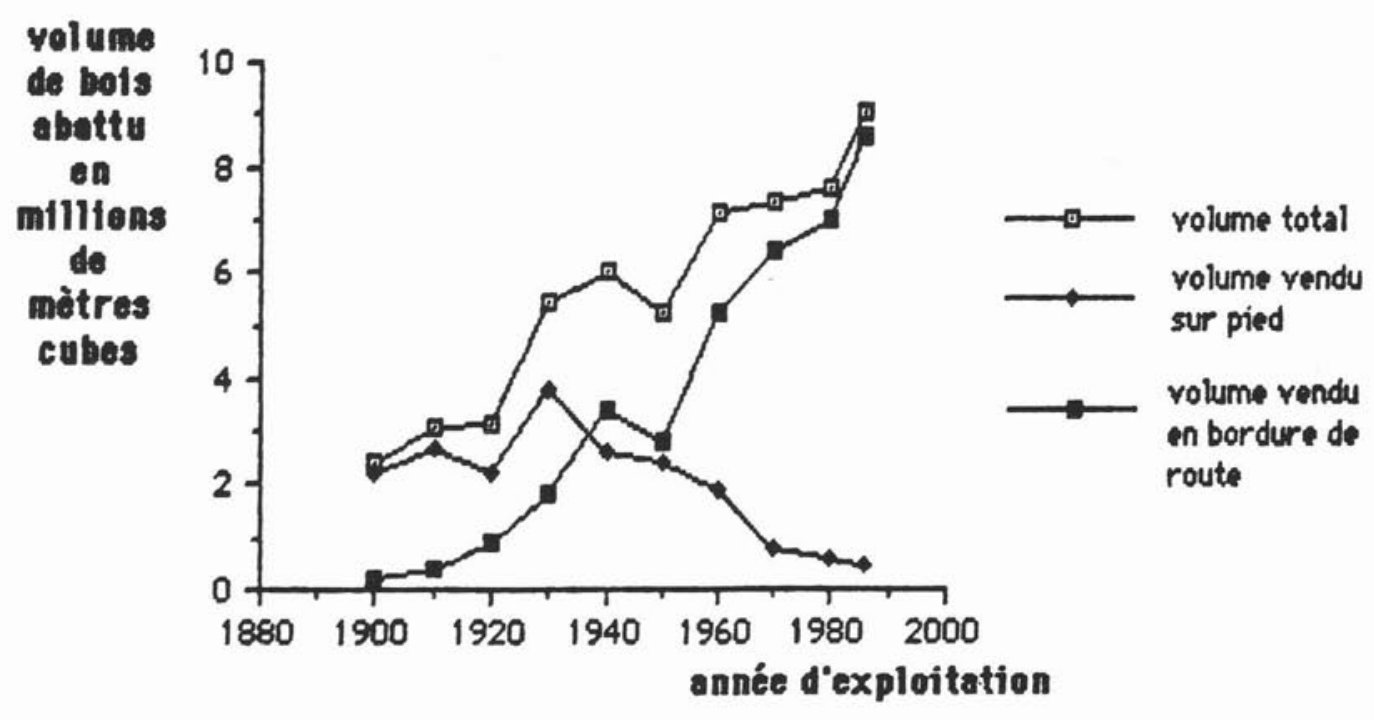

Figure 2. Répartition des bois abattus sur le domaine public suédois au cours des quatrevingt dernières années.

Les transactions, dont les prix sont contestés, concernent des tiges provenant de forêts primitives. Leur production est donc en apparence nette de coûts. Toutefois, le sol forestier est un facteur de production qui doit être rémunéré même quand les arbres qui y croissent n'ont requis aucun soin particulier de la part de son propriétaire. Une rente correspond au montant de cette rémunération. Les économistes parlent de rente quand une ressource durable est utilisée dans une fonction de production. En d'autres termes, la rente est le coût d'un facteur de production dont la disponibilité est indépendante du marché. C'est le cas de la capacité productive d'un sol forestier. La définition du concept de rente laisse donc comprendre toute la difficulté qu'il y a à tenter de la cerner dans la réalité.

L'expression de la durabilité en termes économiques est ardue. Les administrations publiques préfêrent contourner son évaluation en décrétant sa valeur de façons qui apparaissent aléatoires. C'est là où le mécanisme des enchères peut intervenir afin de rapprocher les transactions affectant la matière ligneuse des terres publiques de la vrai valeur des bois. Toutefois, l'expérience américaine montre que l'affaire est semée d'écueils.

La difficulté semble provenir du fait que les frais préparation d'une enchère, c'est-à-dire une foule de tâches allant des inventaires jusqu'au martelage en passant par le calcul de possibilité, sont noyés dans les coûts de fonctionnement du U.S. Forest Service. Il en irait de même pour les coûts inhérents à la protection de la ressource, à la construction et l'entretien du réseau routier ainsi qu'à l'organisation de la vente. Conséquemment, la mise à prix de la matière ligneuse dans les forêts nationales américaines fausserait les indications résultant d'enchères publiques. Envisager l'application d'enchères pour allouer les bois d'une forêt publique n'est donc pas une condition suffisante pour prétendre à un fonctionnement adéquat du marché.

Sommairement, nous pouvons affirmer que jouer le jeu du marché en forêt publique nécessite la satisfaction de 
trois exigences. Premièrement, l'État doit être conscient de la valeur des intrants nécessaires à la production des bois sur pied. À cet égard, il pourrait établir que le seuil minimal de la rente pour l'usage d'une forêt équivaut au coût de remplacement des peuplements forestiers. Deuxièmement, l'État doit se prévaloir de ses droits de propriété en tant que tels. L'impulsion bénéfique des forces du marché n'apparaitrait donc possible que quand l'État se conduit en propriétaireentrepreneur d'une ressource qu'il a contribué à disponibiliser. Enfin, la part du territoire sous tenure publique ne doit pas dépasser un certain plafond, sinon il y a risque de voir l'État user de sa position comme puissance publique pour tenter de nier les signaux du marché.

\section{Message pour le Québec}

À première vue, nous pourrions conclure que le Québec n'est pas tout à fait prêt à jouer le jeu du marché en foresterie. Les propriétaires privés détiennent la portion congrue des forêts de la province. Quant à lui, le domaine public souffre de l'absence d'un intervenant nettement investi du rôle de propriétaire-entrepreneur. Les autorités s'en remettent plutôt à l'industrie manufacturière pour aménager la forêt.

Pourtant gardons-nous de dénier toute pertinence au régime forestier adopté à la fin de 1986 simplement parce qu'il s'appuie sur des contrats à long terme avec l'industrie de transformation. Un tel jugement est injuste. L'irruption du Québec en sylviculture est trop récente. Le monde forestier aborde à peine une période de transition entre l'exploitation d'une forêt primitive et l'utilisation d'une forêt issue d'efforts sylvicoles. Au fur et à mesure qu'une entreprise de production forestière succèdera à une entreprise d'extraction dont le comportement est calqué sur celui du secteur minier, les forces du marché s'exerceront et modifieront la nature des intervenants. La forêt cultivée a un coût incontournable que la raréfaction de la matière ligneuse justifiera tout en diversifiant les formules d'allocation.

Évidemment la rareté relative des approvisionnements en bois comporte plusieurs effets qui tempèrent tout débordement d'optimisme. Cependant, même si elle stimule des changements technologiques et favorise la substitution de la matière ligneuse comme facteur de production, la rareté des bois haussera immanquablement le prix à payer pour la matière ligneuse. Ce dernier phénomène mettra en valeur la fonction de producteur forestier au même titre que celle du transformateur.

Le besoin de créer un secteur étatique témoin et surtout de faire apparaître une véritable classe de producteurs forestiers iront de pair. Certains indices annoncent ce scénario. La Loi sur les forêts ne prévoit-elle pas l'implantation de réserves forestières ainsi que l'octroi d'une priorité aux productions forestières privées? Les outils pour jouer le jeu du marché en parallèle des contrats à long terme avec les entreprises de transformation sont donc déjà disponibles. Reste à savoir si l'esprit de la Loi est conforme à sa lettre.

Dans une veine prospectiviste, une redéfinition de la tenure forestière semble souhaitable. Le partage actuel des terres forestières entre l'État et les propriétaires privés apparait inadéquat. Le domaine public du Québec est trop vaste. Les autorités devraient envisager l'opportunité d'une privatisation du territoire. Nous sommes très conscients que parler de privatiser la forêt publique, c'est ouvrir une boîte de Pandore. Cependant, nous ne sommes plus au XIX ${ }^{\mathrm{e}}$ siècle.
L'environnement décisionnel n'est plus dominé par la naïve croyance que l'intensité du jeu concurrentiel suffit à obtenir une utilisation efficace des ressources productives de la société et par la même occasion, à livrer une production efficiente pour cette société.

La complexité des relations entre une société et son environnement biophysique oblige des actions législatives et réglementaires de la part de l'État. L'allocation de droits de propriété sur le domaine public est désirable à la seule condition que les pouvoirs publics prennent des dispositions pour que les nouveaux propriétaires respectent des objectifs de continuité du milieu forestier, de perpétuation des établissements humains qui y sont arrimés et de protection des propriétés environnementales de la forêt. Autrement dit, il s'agit de s'assurer que les propriétaires de la forêt se comportent en propriétaire-entrepreneur et non en agioteur.

Pour reprendre les mots de Peter Drucker (1989), qui n'est pas particulièrement un «vert», le défi des années qui viennent consiste à comprendre que la performance d'une entreprise ne se mesure pas uniquement en termes de dividendes mensuels et annuels ou dans un rapport boursier cours/ bénéfice le plus petit possible. L'indice d'une bonne performance se cache plutôt dans les opportunités d'emplois durables liées à l'activité de l'entreprise et par la capacité de cette dernière à créer une richesse équilibrée entre le court et le long terme. Pour le secteur forestier, réconcilier l'environnement et l'économie implique une nouvelle dynamique entre le secteur privé et l'État. Elle se fonde sur l'idée, somme toute triviale, que le futur a une valeur en dépit des résultats obtenus par les techniques d'actualisation.

\section{En conclusion}

Le secteur forestier québécois nous semble en bonne posture pour profiter de l'influence des forces du marché. Mais, des facteurs propres au contexte du Québec empêchent l'aboutissement de ces forces vers une situation où la production forestière représente un centre de profits. Les pouvoirs publics contrôlent une part trop grande des superficies forestières de la province. De plus, la confusion des genres entre l'État définisseur de rapports sociaux et l'État propriétaire-entrepreneur nuit à la gestion du domaine public. Enfin, l'ambiguité du concept de rente altère l'action du marché quand il est appliqué à une ressource héritée du seul passage du temps sur un territoire.

Cependant, il apparait que la rareté relative de la matière ligneuse dans la problématique québécoise est porteuse de changements. Tôt ou tard, les intervenants évoluant sur le domaine public seront amenés à passer le test du marché comme producteurs forestiers. De son côté, l'État sera appelé à mieux asseoir son rôle de propriétaire-entrepreneur. L'avènement de la sylviculture et d'un État véritablement propriétaire rendront judicieuses les intentions formulées dans la Loi sur les forêts au sujet de la création de réserves forestières et de la valorisation des productions forestières privées.

\section{Références}

Appelroth, S.E. 1984. "Forest Policy and Law in Finland" in Lortie, M. (ed.) Forest ressources management - The influence of policy and law, 1984 International Forest Congress, pp. 29-31. 
Campbell, J. 1988. "British forestry - An international perspective" in Commonwealth forestry review, Vol. $67 \mathrm{~N}^{\circ} 2$, pp. 115-128.

Davis, L.K. and K.N. Johnson, 1987. Forest Management. Third edition, McGraw-Hill Book Company, pp. 1-26.

Drucker, P., 1989. "The futures that have already happened" in The Economist, Vol. $313 \mathrm{~N}^{\circ} 625$, pp. 19-24.

Emerson, P., A.T. Stout and D. Kloepfer, 1984. "The Feds can't see their losses in the trees" in Wall Street Journal, VOL. CCIV $\mathrm{N}^{\circ} 96$, édition du 14 novembre, p. 32.

Hermelin, J. 1986. "Comparing scandinavian and canadian forestry practices"' in Martin D. ed. (1988). Small scale forestry; experience and potential, pp. 20-34.

Hirvonen, R., 1984. La foresterie en Finlande, Environnement Canada, Service canadien des forêts, DPC-X-16F, 53 p.
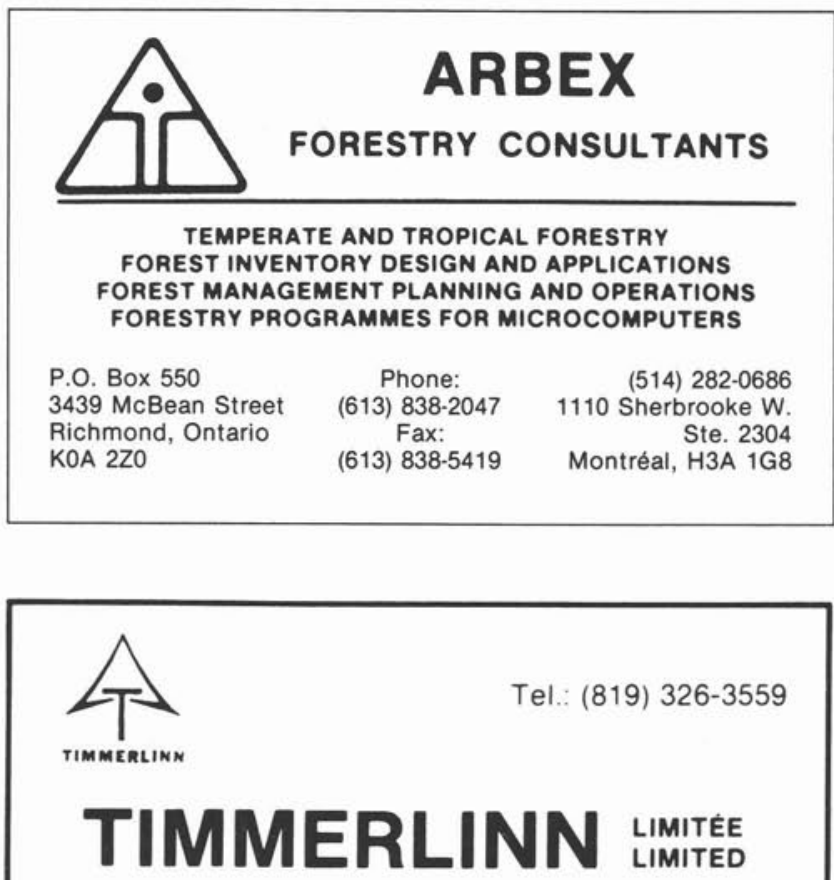

PROFESSIONAL AND TECHNICAL SERVICES IN FORESTRY AND ENVIRONMENTAL SCIENCES

R.R. No. 2, STE. AGATHE DES MONTS, QUE. J8C $2 Z 8$

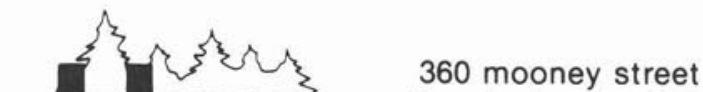 thunder bay, ontario P7B 5R4}

FOREST RESOURCE

- Inventory, Surveys and Evaluations

\section{REFORESTATION EQUIPMENT \\ - Sales Service Parts Repair}

REGENERATION \& SITE PREPARATION

- Contract Assessment Consulting

Tele 807-344-0811 Fax 807-345-3440

Toll free 1.800-465-3001

Hofsten, von E., 1980. Histoire du Domänverket, Domänkoncernen, $36 \mathrm{p}$.

Johnston, D.R., A.J. Grayson, and R.T. Bradley. 1967. Forest Planning, Faber and Faber Limited, pp. 58-86.

Koljonen, K., 1986. "Employment effects of small-scale private forestry" in Martin D. ed. (1988) Small scale forestry; experience and potential pp. 94-107.

Ministère de l'Énergie et des Ressources, 1989. Ressource et industrie forestières, Portrait statistique 1988, pp. 12 et 23.

Price, C., 1989. The Theory and Application of Forest Economics, Basil Blackwell, pp. 350-365.

Sydow, von H., 1985. "Safeguarding the timber supply" in Moving Downstream: Canada's Strategy for World Market Success, Financial Post Conference, pp. 30-33.

\section{EDWARD S. FELLOWS}

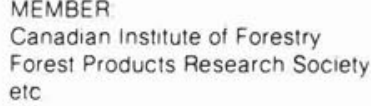

Canadian Institute of Forestry Forest Products Research Society etc

\section{FORESTRY \& FOREST PRODUCTS CONSULTANT}

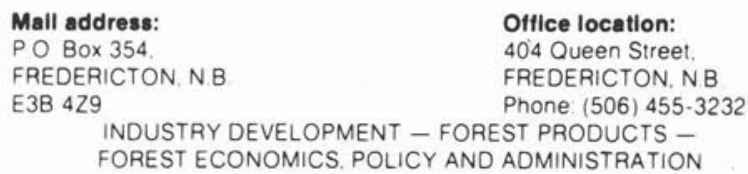

\section{Woodlot Service (1978) Ltd.}

"All Matters Pertalning to Forestry"

GORDON B. YOUNG, B.Sc.F., M.F. Registered Professional Forester

\section{DAISHOWA}

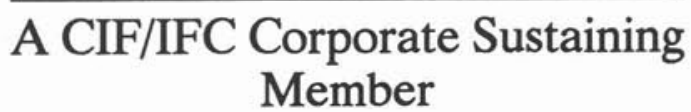

